\title{
Consumo de fuentes de hierro y niveles de hemoglobina - IV ciclo de la E.P de enfermería de la Universidad Privada de Huancayo Franklin Roosevelt. Huancayo 2018
}

Consumption of iron sources and hemoglobin levels - IV cycle of the nursing E.P of the Universidad Privada de Huancayo Franklin Roosevelt. Huancayo 2018

\author{
María Del Carmen Pilco Villagra ${ }^{1}$, Darwin Rivas Alarcón
}

\section{RESUMEN}

Objetivos: Determinar la relación que existe entre el consumo de fuentes de hierro y niveles de hemoglobina en estudiantes del IV ciclo de la escuela profesional de enfermería de la Universidad Roosevelt. Huancayo 2018. Material y Métodos: Es una descripción de dos variables individuales que tienen relación de tipo correlacional y que no se miden en el tiempo. Resultados: De acuerdo a los resultados obtenidos con Rho $=0,483$, existe correlación moderada con una relación considerable entre el consumo de fuentes de hierro y niveles de hemoglobina. Hay una correlación moderada entre el consumo de fuentes de hierro hemínico y los niveles de hemoglobina, Rho = 0,512. Existe correlación moderada entre el consumo de fuentes de hierro no hemínico y los niveles de hemoglobina, Rho $=0,478$. Existe correlación ligera entre el consumo de inhibidores de la absorción del hierro y los niveles de hemoglobina, Rho $=0,151$. Existe una correlación baja entre el consumo de fuentes de vitamina $\mathrm{C}$ y los niveles de hemoglobina, Rho $=0,347$.

PALABRAS CLAVE: Consumo de fuentes de Hierro, hemoglobina.

\section{SUMMARY}

Objectives: To determine the relationship between the consumption of iron sources and hemoglobin levels in students of the fourth cycle of the professional nursing school of Roosevelt University. Huancayo 2018. Material and Methods: It is a description of two individual variables that have a correlational relationship and that are not measured in time. Results: According to the results obtained with Rho $=0.483$, there is a moderate correlation with a considerable relationship between the consumption of iron sources and hemoglobin levels. There is a moderate correlation between the consumption of heminic iron sources and hemoglobin levels, Rho $=0.512$. There is a moderate correlation between the consumption of non-heminic iron sources and hemoglobin levels, Rho $=0.478$. There is a slight correlation between the consumption of iron absorption inhibitors and hemoglobin levels, Rho $=$ 0.151 . There is a low correlation between the consumption of sources of vitamin $\mathrm{C}$ and hemoglobin levels, $\mathrm{Rho}=$ 0.347 .

KEYWORDS: Consumption of sources of iron, hemoglobin 


\section{INTRODUCCIÓN}

Según la Organización Mundial de la Salud (OMS), aproximadamente 2 millones de personas en el mundo sufren de anemia por deficiencia de hierro, y la población más vulnerable son los niños pequeños y las gestantes (1). En el Perú, según la Encuesta Demográfica y de Salud Familiar (ENDES) del Instituto Nacional de Estadística e Informática la prevalencia de anemia en niños menores de 5 años alcanza el 46,2 \% y en niños menores de 2 años es mayor (66\%) (2). Estos datos relevantes conllevan a sugerir que los estudiantes de ciencias de la salud, especialmente los futuros enfermeros, debieran tener adecuados niveles de hemoglobina, que demuestren que no sólo desarrollan la parte teórica, más por el contrario la práctica de una buena nutrición es importante, contribuyendo a la formación de profesionales capacitados que sean capaces de adoptar estrategias varias que permitan disminuir estos niveles de anemia en poblaciones vulnerables. Los efectos de la anemia ferropénica resultan trascendentales, sus consecuencias en el desarrollo de capacidades intelectuales y emocionales son determinantes. Los estudiantes de salud deben de practicar buenos hábitos alimentarios con el consumo de alimentos que son fuentes de hierro hemínico, con el fin de evitar la presencia de anemia.

El propósito de la investigación fue determinar la relación que existe entre el consumo de fuentes de hierro y niveles de hemoglobina en estudiantes del IV ciclo de la escuela profesional de enfermería de la Universidad Roosevelt. Huancayo 2018.

\section{MATERIAL Y MÉTODOS}

En el estudio de investigación se aplicó la técnica de la encuesta con el instrumento cuestionario para la variable consumo de fuentes de hierro y para la variable niveles de hemoglobina se utilizó el análisis por hemocontrol, el procesamiento de datos SPSS v. 20. La investigación basada en el método científico, no experimental, es una descripción de dos variables individuales que tienen relación de tipo correlacional donde no se mide las variables en el tiempo o su comportamiento. Según Hernández, Fernández y Baptista, los estudios correlacionales permiten conocer la relación que existe entre dos o más variables en un contexto peculiar, logran medir el grado de asociación entre las variables (3).

\section{RESULTADOS}

Análisis e interpretación sobre las dimensiones de la variable consumo de fuentes de hierro.

En la tabla 1, se dilucida que $9(12,9 \%)$ de los estudiantes encuestados consumen $01 \mathrm{vez}$ a la semana fuentes de hierro hemínico; por otro lado, $43(61,4 \%)$ consumen dos veces por semana las fuentes de hierro regular, $18(25,7 \%)$ de los estudiantes consumen fuentes de hierro con una frecuencia interdiaria respectivamente.

\section{Análisis e interpretación sobre las dimensiones de la variable niveles de hemoglobina.}

En la tabla 2, se observó que $1(1,4 \%)$ de los estudiantes presentan anemia moderada; 4 (5,7\%) presentan anemia leve; por otro lado, 65 (92,9\%) tienen niveles de hemoglobina normales.

\section{Consumo de fuentes de Hierro hemínico}

En la tabla 3 observamos que $5(18,6 \%)$ de los estudiantes encuestados tienen un buen nivel de consumo de hierro hemínico; además 52 (74,3\%) de los estudiantes tienen un regular nivel de consumo de fuentes de hierro hemínico, mientras que 13 (18,6\%) estudiantes tiene un mal consumo de fuente de hierro hemínico.

\section{Consumo de fuentes de Hierro No hemínico}

En la tabla 4 observamos que $16(22,9 \%)$ de estudiantes tienen un nivel de consumo malo de fuentes de hierro No hemínico; de otro lado, se observa que $51(72,9 \%)$ de los estudiantes encuestados refieren que tienen un nivel regular de consumo de fuentes

Tabla 1. Niveles de percepción de la variable: Consumo de hierro

\begin{tabular}{lcc}
\hline Niveles & Frecuencia & Porcentaje \\
\hline Una vez por semana & 9 & 12,9 \\
Dos veces por semana & 43 & 61,4 \\
Interdiario & 18 & 25,70 \\
TOTAL & 70 & 100,00 \\
\hline
\end{tabular}

Tabla 2. Niveles de Hemoglobina

\begin{tabular}{lcc}
\hline Clasificación & Frecuencia & Porcentaje \\
\hline Anemia moderada & 1 & 1,4 \\
Anemia leve & 4 & 5,7 \\
Normal & 65 & 92,9 \\
TOTAL & 70 & 100,00 \\
\hline
\end{tabular}


Tabla 3. Consumo de fuentes de hierro hemínico

$\begin{array}{lcc}\text { Nivel } & \text { Frecuencia } & \text { Porcentaje } \\ \text { Bueno } & 5 & 7,1 \\ \text { Regular } & 52 & 74,3 \\ \text { Malo } & 13 & 18,6 \\ \text { TOTAL } & 70 & 100,00\end{array}$

Tabla 4. Niveles de consumo de fuentes de hierro No hemínico

\begin{tabular}{lcc}
\hline & Frecuencia & Porcentaje \\
\hline Bueno & 16 & 22,9 \\
Regular & 51 & 72,9 \\
Malo & 3 & 4,3 \\
TOTAL & 70 & 100,00 \\
\hline
\end{tabular}

de hierro No hemínico, se observa que 3 (4,3\%) estudiantes tiene un buen nivel de consumo de fuentes de hierro No hemínico.

Consumo de inhibidores de la absorción de hierro.

En la tabla 05, observamos que $54(77,1 \%)$ de los estudiantes consumen alimentos que tienen inhibidores de la absorción del hierro, además 15 (21,4\%) de los estudiantes encuestados muestran un consumo regular de alimentos inhibidores de la absorción del hierro, finalmente $1(1,4 \%)$ de los estudiantes muestran un consumo bueno de alimentos inhibidores de hierro.

\section{Consumo de fuentes de vitamina $C$}

En la tabla 6 observamos que $25(35,7 \%)$ estudiantes tienen un consumo malo de fuentes de vitamina C, 38 $(54,3 \%)$ de los estudiantes tienen un consumo regular de fuentes de vitamina $\mathrm{C}$, finalmente se observa que 7 $(10 \%)$ estudiantes encuestados tiene un buen consumo de alimentos que son fuente de vitamina $\mathrm{C}$.

Relación entre fuentes de hierro y niveles de hemoglobina: Existe relación significativa entre el consumo de fuentes de hierro y niveles de hemoglobina en estudiantes del IV ciclo de la escuela profesional de enfermería de la universidad Roosevelt. Huancayo 2018.

\section{Hipótesis estadística}

Ho: No existe relación significativa entre el consumo de fuentes de hierro y niveles de hemoglobina en estudiantes del IV ciclo de la escuela profesional de enfermería de la universidad Roosevelt. Huancayo 2018.

H1: Existe relación significativa entre el consumo de fuentes de hierro y niveles de hemoglobina en estudiantes del IV ciclo de la escuela profesional de enfermería de la universidad Roosevelt. Huancayo 2018.

Nivel de significancia: El nivel de significación teórica es $\alpha=0,05$. Que corresponde a un nivel de confiabilidad del $95 \%$

Regla de decisión: El nivel de significancia “p” es menor que $\alpha$, rechazar Ho. El nivel de significancia " $p$ " no es menor que $\alpha$, no rechazar Ho

Prueba estadística: Rho de Spearman

Decisión estadística: Debido a que $\mathrm{p}=0,000$ es menor que 0,05 se rechaza la Ho.

Conclusión: De acuerdo a los resultados obtenidos $($ Rho $=0,483 ; p=0,000$ menor que 0,05$)$, existe correlación moderada con una relación considerable entre el consumo de fuentes de hierro y niveles de hemoglobina consecuentemente, se rechaza la hipótesis nula.

Tabla 5. Niveles de consumo de inhibidores de la absorción de hierro

\begin{tabular}{lcc}
\hline Nivel & Frecuencia & Porcentaje \\
\hline Malo & 54 & 77,1 \\
Regular & 15 & 21,4 \\
Bueno & 1 & 1,4 \\
TOTAL & 70 & 100,00 \\
\hline
\end{tabular}

Tabla 6. Niveles de consumo de fuentes de vitamina $\mathrm{C}$

\begin{tabular}{lcc}
\hline Nivel & Frecuencia & Porcentaje \\
\hline Malo & 5425 & 35,7 \\
Regular & 38 & 54,3 \\
Bueno & 7 & 10 \\
TOTAL & 70 & 100,00 \\
\hline
\end{tabular}

El consumo de hierro hemínico y los niveles de hemoglobina

\section{Hipótesis específica de la investigación}

Existe relación significativa entre el consumo de fuentes de hierro hemínico y niveles de hemoglobina en estudiantes del IV ciclo de la escuela profesional de enfermería de la Universidad Roosevelt. Huancayo 2018.

\section{Hipótesis estadística}

Ho: No existe relación significativa entre el consumo de hierro y los niveles de hemoglobina en estudiantes del IV ciclo de la escuela profesional de enfermería de la universidad Roosevelt. Huancayo 2018. 
H1: Existe relación significativa entre el consumo de hierro y los niveles de hemoglobina en estudiantes del IV ciclo de la escuela profesional de enfermería de la universidad Roosevelt. Huancayo 2018.

Nivel de significancia: El nivel de significación es $\alpha$ $=0,05$

Que corresponde a un nivel de confiabilidad del 95\%. Regla de decisión: El nivel de significancia " $p$ ” es menor que $\alpha$, rechazar Ho.

El nivel de significancia " $p$ " no es menor que $\alpha$, no rechazar Ho

Prueba estadística: Rho de Spearman

Decisión estadística: Debido a que $\mathrm{p}=0,000$ es menor que 0,05 se rechaza la Ho.

Conclusión: De acuerdo a los resultados obtenidos (Rho $=0,512, p=0,000$ menor que 0,05 ), el consumo de fuentes de hierro hemínico se relaciona con los niveles de hemoglobina (correlación moderada, relación considerable); entonces se rechaza la hipótesis nula.

\section{Consumo de fuentes de hierro no hemínico y los niveles de hemoglobina}

\section{Hipótesis especifica de la investigación}

Existe relación significativa entre el consumo de fuentes de hierro no hemínico y los niveles de hemoglobina en los estudiantes del IV ciclo de la escuela profesional de Enfermería de la Universidad Roosevelt. Huancayo 2018.

Hipótesis estadística

Ho: No existe relación significativa entre el consumo de fuentes de hierro no hemínico y los niveles de hemoglobina en los estudiantes del IV ciclo de la escuela profesional de Enfermería de la Universidad Roosevelt. Huancayo 2018

H1: Existe relación significativa entre el consumo de fuentes de hierro no hemínico y los niveles de hemoglobina en los estudiantes del IV ciclo de la escuela profesional de Enfermería de la Universidad Roosevelt. Huancayo 2018

Nivel de significancia

El nivel de significancia teórica es $\alpha=0,05$.

Que corresponde a un nivel de confiabilidad del 95\% Regla de decisión

El nivel de significancia " $p$ ” es menor que $\alpha$, rechazar Ho.

El nivel de significancia " $p$ " no es menor que $\alpha$, no rechazar Ho.

Prueba estadística: Rho Spearman

Decisión estadística: Debido a que $\mathrm{p}=0,000$ es menor que 0,05 se rechaza la Ho.

Conclusión: De acuerdo a los resultados obtenidos
( $R$ ho $=0,478, p=0,000$ menor que 0,05$)$, el consumo de fuentes de hierro no hemínico se relaciona considerable con los niveles de hemoglobina; entonces, se rechaza la hipótesis nula

\section{Consumo de los inhibidores de la absorción de hierro y los niveles de hemoglobina}

\section{Hipótesis especifica de la investigación}

Existe relación significativa entre el consumo de los inhibidores de la absorción de hierro y los niveles de hemoglobina en los estudiantes del IV ciclo de la escuela profesional de Enfermería de la Universidad Roosevelt. Huancayo 2018.

Hipótesis estadística

Ho: No existe relación significativa entre el consumo de los inhibidores de la absorción de hierro y los niveles de hemoglobina en los estudiantes del IV ciclo de la escuela profesional de Enfermería de la Universidad Roosevelt. Huancayo 2018

H1: Existe relación significativa entre el consumo de los inhibidores de la absorción de hierro y los niveles de hemoglobina en los estudiantes del IV ciclo de la escuela profesional de Enfermería de la Universidad Roosevelt. Huancayo 2018.

Nivel de significancia

El nivel de significancia teórica es $\alpha=0,05$

Que corresponde a un nivel de confiabilidad del 95\%

Regla de decisión

El nivel de significancia " $p$ ” es menor que $\alpha$, rechazar Ho.

El nivel de significancia " $p$ " no es menor que $\alpha$, no rechazara Ho.

Prueba estadistica: Rho Spearman

Decisión estadística: Debido a que $\mathrm{p}=0,213$ es mayor que 0,05 se acepta la Ho.

Conclusiones: De acuerdo a los resultados obtenidos ( Rho $=0,151, p=0,213$ mayor que 0,05$)$, el consumo de los inhibidores de la absorción de hierro tiene correlación ligera con los niveles de hemoglobina; consecuentemente, se acepta la hipótesis nula. Existe relación significativa entre el consumo de fuentes de vitamina $\mathrm{C}$ y los niveles de hemoglobina

Hipótesis específica de la investigación: Existe relación significativa entre el consumo de fuentes de vitamina $\mathrm{C}$ y los niveles de hemoglobina en estudiantes de IV ciclo de la escuela profesional de enfermería de la Universidad Roosevelt. Huancayo 2018.

Hipótesis estadística:

Ho: No existe relación significativa entre el consumo de fuentes de vitamina $\mathrm{C}$ y los niveles de hemoglobina en estudiantes de IV ciclo de la escuela profesional de enfermería de la Universidad Roosevelt. Huancayo 
2018.

H1: Existe relación significativa entre el consumo de fuentes de vitamina $\mathrm{C}$ y los niveles de hemoglobina en estudiantes de IV ciclo de la escuela profesional de enfermería de la Universidad Roosevelt. Huancayo 2018.

Nivel de significancia: El nivel de significancia teórica es $\alpha=0,05$

Que corresponde a un nivel de confiabilidad del 95\% Regla de decisión: El nivel de significancia "p" es menor que $\alpha$, rechazar Ho.

El nivel de significancia "p" no es menor que $\alpha$, no rechazar la Ho.

Prueba estadística: Rho Spearman

Decisión estadística: Debido a que $\mathrm{p}=0,000$ es menor que 0,05 se rechaza la Ho.

Conclusión: De acuerdo a los resultados (Rho $=$ $0,347, p=0,000$ menor que 0,05 ), el consumo de fuentes de vitamina $\mathrm{C}$ se relaciona con los niveles de hemoglobina (correlación baja); en consecuencia, se rechaza la hipótesis nula.

\section{DISCUSIÓN}

De acuerdo al objetivo general y después de realizar las pruebas estadísticas de Rho de Spearman se concluye que el consumo de fuentes de hierro se relaciona significativamente con niveles de hemoglobina en cada una de las dimensiones descritas; respecto a la hipótesis general y según los resultados que se hallaron por Rho de Spearman $=0,512 \mathrm{y}$ con nivel de significancia $\mathrm{p}=0,000$ que es menor al nivel de significancia teórica de $\alpha=0,05$ se acepta la hipótesis planteada y se afirma que existe una correlación moderada con una relación considerable entre las dos variables; entonces se concluye que a mayor consumo de fuentes de hierro mejores niveles de hemoglobina; estos resultados finales concuerdan con los hallados por Choque, quien determinó la prevalencia de anemia y consumo de alimentos fuentes de hierro (4).

\section{CONCLUSIONES}

Se determinó que existe correlación moderada con una relación considerable entre las variables de consumo

Tabla 7. Correlación entre consumo de hierro y niveles de hemoglobina

\begin{tabular}{lllll}
\hline & & Consumo de hierro & Niveles de $\mathrm{Hb}$ \\
\hline & \multirow{3}{*}{$\begin{array}{l}\text { Consumo de hierro } \\
\text { Rho de }\end{array}$} & Coeficiente de correlación & 1,000 & $0,483^{* *}$ \\
Spearman & Sig. (bilateral) & & 0,000 \\
& & $\mathrm{~N}$ & 70 & 70 \\
& Hemoglobina & Coeficiente de correlación & $0,483^{* *}$ & 1,000 \\
& Sig. (bilateral) & 0,000 & \\
\hline Fuente: Base de datos (SPSS 24) & $\mathrm{N}$ & 70 & 70
\end{tabular}

Tabla 8. Correlación entre el consumo de fuentes de hierro hemínico y los niveles de hemoglobina

\begin{tabular}{lllll}
\hline & & $\begin{array}{l}\text { Fuentes de } \\
\text { hierro hemínico }\end{array}$ & $\begin{array}{l}\text { Niveles de } \\
\text { Hemoglobina }\end{array}$ \\
\hline & & Coeficiente de correlación & 1,000 & $0,512^{* *}$ \\
& $\begin{array}{l}\text { Fuentes de hierro } \\
\text { hemínico }\end{array}$ & Sig. (bilateral) & & 0,000 \\
$\begin{array}{l}\text { Rho de } \\
\text { Spearman }\end{array}$ & & $\mathrm{N}$ & 70 & 70 \\
& & Coeficiente de correlación & $0,512^{* *}$ & 1,000 \\
& $\begin{array}{l}\text { Niveles de } \\
\text { Hemoglobina }\end{array}$ & Sig. (bilateral) & 0,000 & \\
& & $\mathrm{~N}$ & 70 & 70 \\
\hline
\end{tabular}


Tabla 9. Correlación entre existe relación significativa entre el consumo de fuentes de hierro no hemínico y los niveles de hemoglobina

\begin{tabular}{lllll}
\hline & & & $\begin{array}{l}\text { Consumo fuentes } \\
\text { de hierro no hem }\end{array}$ & $\begin{array}{l}\text { Niveles de } \\
\text { hemoglobina }\end{array}$ \\
\hline & $\begin{array}{l}\text { Consumo de fuentes } \\
\text { de hierro no hem }\end{array}$ & Coeficiente de correlación & 1,000 & 0,478 \\
& & Sig. (bilateral) & 0000 \\
$\begin{array}{l}\text { Rho de } \\
\text { Spearman }\end{array}$ & & N & 70 & 70 \\
& Niveles de Hb & Coeficiente de correlación & $0,478^{* *}$ & 1,000 \\
& & Sig. (bilateral) & 0,000 & \\
& & $\mathrm{~N}$ & 70 & 70 \\
\hline
\end{tabular}

Tabla 10. Correlación entre el consumo de los inhibidores de la absorción de hierro y los niveles de hemoglobina

\begin{tabular}{lllcc}
\hline & & Inhibidores & Niveles de hemoglobina \\
\hline & \multirow{3}{*}{ Inhibidores } & Coeficiente de correlación & 1,000 & $0,151^{* *}$ \\
& & Sig. (bilateral) & & 0,213 \\
Rho de & & $\mathrm{N}$ & 70 & 70 \\
Spearman & & Coeficiente de correlación & $0,151^{* *}$ & 1,000 \\
& Niveles de & Sig. (bilateral) & 0,213 & \\
& hemoglobina & $\mathrm{N}$ & 70 & 70 \\
\hline
\end{tabular}

Tabla 11. Correlación entre el consumo de fuentes de vitamina $\mathrm{C}$ y los niveles de hemoglobina

\begin{tabular}{lllll}
\hline & & Fuentes de vit C & Niveles de hemoglobina \\
\hline & & Coeficiente de correlación & 1,000 & $0,347^{* *}$ \\
\multirow{3}{*}{$\begin{array}{l}\text { Rho de } \\
\text { Spearman }\end{array}$} & & & 0,003 \\
& & N & 70 & 70 \\
& Niveles de & Coeficiente de correlación & $0,347^{* *}$ & 1,000 \\
& hemoglobina & Sig. (bilateral) & 0,003 & \\
& & $\mathrm{~N}$ & 70 & 170 \\
\hline
\end{tabular}

de hierro y niveles de hemoglobina en los estudiantes, donde se determinó la Rho de Spearman $=0,483$ y nivel de significancia teórica de $\alpha=0,00$, aceptándose la hipótesis planteada (tabla 7).

Se determinó en el estudio de investigación una correlación moderada entre el consumo de fuentes de hierro hemínico y los niveles de hemoglobina, donde de acuerdo a la Rho de Spearman de 0,512 y con $p=$ 0,000 que es menor a 0,05 ; por lo tanto, se acepta la hipótesis planteada (tabla 8).
Se determinó en el presente estudio que existe una correlación moderada entre el consumo de fuentes de hierro no hemínico y los niveles de hemoglobina, donde se tiene una Rho de Spearman de 0,478 y con $p=0,000$ que es menor a 0,05 ; por ende, se acepta la hipótesis planteada (tnbla 9).

Se determinó que existe correlación ligera entre el consumo de inhibidores de la absorción del hierro y los niveles de hemoglobina, según Rho de Spearman $=0,151$ existe una correlación ligera (tabla 10). 
Se determinó que existe una correlación entre las dimensiones de consumo de fuentes de vitamina $\mathrm{C}$ y los niveles de hemoglobina, con el resultado de Rho $=0,347$ que significa que existe una correlación baja y relación definida pero pequeña, además de un nivel de correlación $\mathrm{p}=0,003$ donde es menor al valor de significancia teórica de $\alpha=0,05$; por tanto, se acepta la hipótesis planteada (tabla 11).

\section{REFERENCIAS BIBLIOGRÁFICAS}

1. Organización Mundial de la Salud. Situación de deficiencia de Hierro. Ginebra: Organización Mundial de la Salud; 2006.
2. Instituto Nacional de Estadística e Informática. Encuesta Demográfica y de salud familiar (ENDES). LÑima: Organización Mundial de la Salud;2012.

3. Hernández R, Fernández C, Baptista P. Metodología de la investigación. Ciudada fr Mexico: McGrawHill; 2006. p.104-105.

4. Choque M. Prevalencia de anemia y consumo de alimentos fuentes de hierro, en mujeres en edad fértil de la I.E.I.B ciudad de El Alto. Tesis de maestría. La Paz, Bolivia: Universidad Mayor de San Andrés; 2016.

Recibido: $\quad 12 / 07 / 2019$

Aceptado: 2/10/2019 